\title{
Pratiques
}

Linguistique, littérature, didactique

$157-158 \mid 2013$

Théories et pratiques des genres

\section{Le Cru et le Lu}

Ethnocritique d'un album pour la jeunesse, Le Géant de Zéralda

Marie-Christine Vinson

\section{(2) OpenEdition}

Journals

Édition électronique

URL : http://journals.openedition.org/pratiques/3856

DOI : $10.4000 /$ pratiques.3856

ISSN : 2425-2042

Éditeur

Centre de recherche sur les médiations (CREM)

Édition imprimée

Date de publication : 1 juin 2013

Référence électronique

Marie-Christine Vinson, « Le Cru et le Lu », Pratiques [En ligne], 157-158 | 2013, mis en ligne le 18

décembre 2017, consulté le 14 novembre 2019. URL : http://journals.openedition.org/pratiques/3856

(c) Tous droits réservés 


\section{LE CRU ET LE LU ${ }^{(*)}$ \\ Ethnocritique d'un album pour la jeunesse, Le Géant de Zéralda}

\section{Marie-Christine Vinson}

Universite de Lorraine/CREM

Le Géant de Zéralda de Tomi Ungerer paraît en 1971 à l'École des loisirs ${ }^{(1)}$. Dans cet album comme dans beaucoup d'autres d'ailleurs, l'illustration occupe une place importante, souvent la pleine page à droite et même sept doubles pages. Les caractéristiques graphiques des personnages se répètent tout au long de l'ouvrage : le bonnet rouge de l'ogre, son grand nez, les yeux ronds et malicieux de la fillette, son sourire. Le texte qui accompagne les images est un conte. Et que dit le conte ? Le destin d'une petite fille, Zéralda l'orpheline : elle vit seule avec son père, sa mère est morte. Un jour elle rencontre un ogre. Grâce à ses talents de cuisinière, elle le transforme en prince charmant et, devenue grande, elle l'épouse et a avec lui beaucoup d'enfants. Le conte, en effet, est « toujours, peu ou prou, un récit exemplaire, ses péripéties désignent la bonne voie, semée d'épreuves nécessaires, et qui aboutit toujours à l'achèvement et à l'installation du jeune héros. Et c'est pour cela que les contes finissent bien ${ }^{(2)}$. »

Mais dans notre album, le monde est-il totalement parfait ? Comment est raconté ce passage qui fait d'un ogre vorace et hirsute un prince parfaitement charmant et d'une petite fille innocente, une jeune femme épanouie?

(*) Cet article est déjà paru in Horizons ethnocritiques, J.-M. Privat et M. Scarpa (éds.), P.U.N., Nancy, 2010, p. 81-96. Nous remercions les P.U.N. et les responsables de la collection EthnocritiqueS de nous avoir aimablement autorisée à reproduire ici cet article.

(1) L'album paraît en 1970 chez Diogenes Verlag à Zurich en Suisse.

(2) Claudine Fabre-Vassas, Daniel Fabre, Du rite au roman. Parcours d'Yvonne Verdier, in Yvonne Verdier, Coutume et Destin. Thomas Hardy et autres essais, NRF Gallimard, 1995, p.30. 


\section{La structure culturelle des lieux imaginaires}

Le monde évoqué dans Le Géant de Zéralda, est un monde traditionnel et idéalisé : on s'éclaire à la bougie, on tire l'eau du puits, on se déplace en charrette, on va au marché vendre les produits de sa récolte, les tenues vestimentaires empruntent au Moyen-Âge comme à un autrefois imprécis. Les lieux dans lesquels évoluent les personnages ne sont pas sans rappeler la division tripartite de l'espace pratique et symbolique des sociétés paysannes occidentales et précapitalistes. Quelle est la fonction de ces différents espaces et de leur configuration dans l'album ?

\subsection{La ville ensauvagée}

L'histoire commence dans une ville, un gros bourg où la domus constitue l'élément essentiel du décor. Les parents y élèvent leurs enfants, les maîtres y instruisent leurs élèves. Replié sur lui-même, enfermé dans ses propres frontières, le monde de la ville a repoussé hors de sa vue tout élément qui rappelle la nature. La flore et la faune sont quasiment exclues. Seuls, un oiseau en cage et une fleur en pot, témoignent encore de ce monde absent.

Cet univers urbain et clos de la pensée domestiquée qui ignore l'univers de la pensée sauvage est perturbé par l'irruption d'un ogre. C'est le retour du refoulé culturel. Pourvu de dents pointues, d'un grand couteau ensanglanté, il a deux yeux féroces et la barbe piquante d'un homme ensauvagé. Ses vêtements laissent voir un torse velu et des bras imposants. Il mange les enfants de préférence et sa faim ne peut être rassasiée. Il habite un château dont on ne voit en page de titre que le pont-levis et la herse d'entrée qui évoque une énorme bouche dentée ! L'ogre vient d'un ailleurs abandonné aux forces sauvages, où les pulsions dévastatrices règnent dans un univers « animalisé », un " saltus ${ }^{(3)}$ »

L'histoire commence donc par un incipit qui met en images et en mots une confrontation brutale et exclusive. Quand l'ogre arrive en ville avec sa démesure, sa voracité, il ensauvage tout sur son passage. L'anthropophagie, comme tout viol de tabou alimentaire, « infecte l'univers » dit Claude Lévi-Strauss et « le laisse en désordre, le rend au chaos », ajoute Yvonne Verdier ${ }^{(4)}$.

Et c'est bien une véritable poussée dé-civilisatrice que déclenche la venue de l'ogre : les parents ne peuvent plus protéger leurs enfants, les écoles sont vides et les maîtres au chômage. Les souterrains, les caves profondes deviennent des refuges recherchés. Le texte le dit, les illustrations le montrent. Deux cosmolo-

(3) Fernand Braudel, L'identité de la France. Espace et Histoire, Arthaud-Flammarion, 1986, p. 123. «Le saltus, c'est dix, cent choses à la fois : des landes, des collines abandonnées à la végétation sauvage; des vignes qui ont cessé d'être cultivées mais où survivent d'ordinaire quelques arbres fruitiers, plantés jadis entre les rangs de ceps, parfois obstinés à produire encore ; ou bien des lignes désordonnées de charmilles, clôtures anciennes qui, abandonnées à elles-mêmes, ont grandi démesurément, aussi hautes que celles qui bordent, sous la main des jardiniers soigneux, les allées princières de 1'Europe de 1'Est... Et plus encore ce sont des formations buissonnantes, " des taillis, des broussailles, entremêlés de végétaux herbacés”. Enfin des forêts, elles surtout».

(4) Yvonne Verdier, "Pour une ethnologie culinaire », L’Homme, Janvier-Mars 1969, n²1, p. 55. 
gies, deux monologies culturelles s'affrontent. Alors les humains s'animalisent. Ils creusent des galeries et se terrent comme des rats. Les habitants de la ville sont submergés par la part animale de l'ogre. Et dans la redistribution des rôles et des catégories qui s'opèrent, ils ne parviennent plus qu'à occuper la place de la proie, du gibier poursuivi : chasseur chassé dans un monde dangereusement mis à l'envers.

Les enfants refoulés dans la partie basse de la maison qui « est le lieu du secret le plus intime à l'intérieur du monde de l'intimité, c'est-à-dire de tout ce qui concerne la sexualité et la procréation ${ }^{(5)} \gg$ doivent refaire, mais de façon inversée, le parcours de leur naissance : au lieu de sortir à la lumière, ils sont enfermés « dans des coffres et des tonneaux, dans des caves sombres et des souterrains ». Mais on peut dire aussi qu'ils sont enterrés vivants. Pour rester en vie, ils doivent en quelque sorte occuper la place des morts, comme un temps de mort symbolique et rituelle...

Ainsi, l'intrusion des forces du «saltus » provoque un retournement qui met le monde à l'envers, sens dessus dessous. Bien sûr ce renversement du monde n'est pas sans rappeler le renversement de Carnaval car notre ogre emprunte ces traits culturels non seulement aux contes traditionnels, mais aussi aux personnages de carnaval, hommes sauvages, ours carnavalesques aux poils hirsutes qui plongent le monde dans une folie temporaire.

\subsection{La petite maison dans la clairière}

À cette configuration conflictuelle et dichotomique qui inverse l'ordre du monde de façon pérenne et non cyclique, répond une autre configuration qui fait coexister, s'articuler les espaces différenciés. Au monde perturbé par l'ogre succède en effet le monde de Zéralda, la petite héroïne de l'histoire. Elle vit dans un lieu protégé, à l'écart, dans « une vallée éloignée » où l'on n'a jamais entendu parler de l'ogre. Le monde de Zéralda s'organise autour de la maison, la domus, qui se trouve dans " une clairière au milieu des bois ». Le père cultive des pommes de terre, du blé, élève des bestiaux. À l'inverse de l'ogre lié au « saltus », le père, cultivateur, est un homme du «campus » qui transforme les espaces incultes en espaces cultivés. La clairière joue à plein son rôle de « clairière culturale ${ }^{(6)} »$ qui organise les espaces en fonction des productions agricoles et qui, du même coup, parvient, en instaurant une zone intermédiaire, à mettre le « saltus » à la bonne distance sans pour autant le supprimer. Effectivement si l'on regarde bien l'image, la maison, l'étable, l'arbre fruitier, le puits se détachent sur le fond sombre de la forêt.

Dans cet univers ternaire bien structuré, Zéralda dont la mère est morte, règne sur la maison et plus particulièrement, sur la cuisine, "cette maison dans la maison ${ }^{(7)} \gg$. L'album accorde une attention particulière à cette pièce dans l'illustra-

(5) Pierre Bourdieu, Esquisse d'une théorie de la pratique, précédé de trois études d'ethnologie kabyle, Paris, Éditions du Seuil, 2000, première parution 1972, p. 66.

(6) Expression citée par Fernand Braudel, op. cit., p. 121.

(7) Emmanuel Le Roy Ladurie, «La domus à Montaillou », Ethnologie Française III, n 1-2, 1973 , p. 47. 
tion. Dans la première double page qui lui est consacrée, le « saltus » et le «campus » entrent dans la maison. Mais ils ne la mettent pas en danger, bien au contraire, ils sont l'un et l'autre domestiqués. Le bois qui vient de la forêt est coupé et rangé prés de la cheminée, les escargots sont en bocaux. Les fruits sont préparés au sirop, mis en conserve : ils témoignent d'une grande maîtrise du temps et permettent d'échapper à la consommation cyclique et saisonnière. Le couteau sur la table s'il évoque le couteau taché de sang de l'ogre présent sur la première page sert ici à découper les carottes en rondelles !

Un peu plus loin dans l'album, l'image d'une autre cuisine s'étale sur une page entière : il s'agit de la cuisine de l'ogre pour qui Zéralda a accepté de travailler. La présence de la petite fille a modifié ce lieu immense et sombre situé dans les profondeurs du château, en pièce accueillante. Le « saltus » y est là aussi parfaitement maîtrisé, civilisé : du gibier (lièvre, canard, faisan) pris à la chasse dont les pièces sont parfaitement alignées, suspendues à des crochets et en attente d'être préparées; le chat lui porte un ruban noué autour du cou et regarde une souris qui ne semble pas craindre d'être dévorée !

Le monde de Zéralda ne fonctionne donc pas à l'exclusion, ne s'organise pas dans un jeu d'oppositions tranchées du sauvage et du familier, mais valorise, dans une certaine mesure, ce que Philippe Descola appelle « la porosité des frontières entre nature et culture ${ }^{(8)} »$. Il s'agit en effet de faire cohabiter « domus », « campus » et « saltus ». Le monde de Zéralda compose avec le civilisé (« domus » et « campus ») et le sauvage (« saltus »), il joue de l'aspect labile des éléments qui passent d'un lieu à un autre et peuvent se « désensauvager ${ }^{(9)}$ ». L'album semble rappeler que la vérité anthropologique des sociétés humaines n'est pas l'opposition manichéenne de la Nature et de la Culture, mais l'indispensable trinité culturelle saisie dans une dynamique toujours singulière.

\subsection{Le Chemin des passages obligés}

Entre le monde de la ville ensauvagée par la présence récurrente de l'ogre et le monde de Zéralda où le « saltus » est «culturalisé », il y a le chemin qui va de la clairière à la ville en passant par la forêt. C'est la zone des passages obligés et des parcours croisés. L'ogre l'emprunte pour se rendre en ville et prélever sa ration d'enfants à croquer. La fillette y circule en charrette pour venir vendre ses produits au marché.

Si elle se trouve sur le chemin, c'est parce qu'à six ans, elle doit sortir du monde de la petite enfance pour grandir. Dans le monde apparemment lissé et policé de la petite maison dans la clairière, il y a une fêlure : Zéralda elle-même. En effet elle occupe un rôle qui n'est pas le sien : elle tient la maison et fait la cuisine comme si elle était l'épouse. Mais comment une fille peut-elle s'épanouir et devenir une femme accomplie si elle vit coupée du reste de la société, en tête-à-tête

(8) Philippe Descola, Par-delà nature et culture, NRF, Éditions Gallimard, 2005, p. 19-57.

(9) Daniel Fabre, «Limites non frontières du Sauvage », L'Homme, Juillet-Décembre 2005, $\mathrm{n}^{\circ} 175-176$ Vérités de la fiction, Éditions de 1’EHESS, p. 427-443. 
avec son père ? Un père ne peut pas garder sa fille pour lui ${ }^{(10)}$, une fille doit apprendre que l'homme avec qui elle peut fonder une famille ne doit appartenir ni à son entourage trop proche, ni en être trop éloigné ${ }^{(11)}$. C'est bien ce problème de distance que le chemin met en scène. En s'y engageant, Zéralda laisse derrière elle un homme trop proche, son père. Le père comme l'ogre partagent des caractéristiques communes que les images mettent en valeur : un bonnet rouge, la moustache, un grand nez, des habits rayés. Ils représentent, à des degrés divers, une force d'ensauvagement qui peut mettre en péril le processus de socialisation de la petite fille. Tous deux sont liés à l'inceste : de façon latente pour le père, de façon patente pour l'ogre cannibale. Or, c'est bien parce qu' on accepte de s'insérer dans un réseau d'interdits et d'obligations, que l'on peut passer de la nature à la culture, de l'animal à l'humain ${ }^{(12)}$.

Certes, Zéralda a la précocité des héroïnes des contes : «A six ans, elle savait déjà faire friture et rôti, bouilli et farce, ragoût et grillade ». Et il semble bien qu'elle maîtrise les feux domestiques (la crémaillère est à bonne distance...). Mais elle ne sait pas tout faire ! Ce qu'elle ne sait pas, c'est « cuire » (faire) les enfants. L'ogre, tout géant qu'il est, doit aussi grandir : coincé dans les marges du « saltus », il est soumis à des appétits sans limites. Il assaisonne les enfants « avec du sel et du poivre », mais sa pratique de l'assaisonnement est mauvaise. Si l'assaisonné est chargé d'une forte connotation sexuelle et lié à l'idée de fécondation $^{(13)}$, la façon de faire de l'ogre est stérile et dévastatrice. La deuxième vérité anthropologique que met donc en évidence, voire que dévoile cette fiction, c'est que les rites d'institution sont en fait des rites de double institution. En effet, l'ogre et Zéralda ont besoin l'un de l'autre et pour que le rite de passage s'accomplisse dans son intégralité, il faut qu'il y ait interaction concomitante entre les deux personnages. Ils se présupposent et s'instituent l'un l'autre, comme, par exemple, charivariseurs et charivarisés. Le travail d'initiation se présente comme une nécessaire co-opération où chacun va apprendre à agir selon le processus de culturation qui lui est propre, même si l'un des deux a l'initiative de l'initiation.

\section{La cuisine de l'écrit}

La question est maintenant de savoir comment Zéralda s'y prend pour avancer sur le chemin du rite, et faire avancer l'ogre avec elle. Certains contes comme le Petit Chaperon rouge parlent de chemin des aiguilles et de chemin des épingles. S'il fallait, ici, nommer le chemin sur lequel s'engagent les deux personnages, on pourrait l'appeler le chemin du cuit et de l'écrit.

(10) Quand, affaibli, alité, il lui demande d'aller au marché à sa place, l'illustration, en vis-àvis, montre, accroché au mur de la chambre, à côté du lit conjugal, un portrait de la mère, barré d'un ruban noir, qui semble en interdire l'accès et signaler la prohibition de l'inceste.

(11) Claude Lévi-Strauss, Le regard éloigné, Paris, Plon, 1983, p. 84 et suivantes. C'est essentiellement dans la partie «Famille, Mariage, Parenté » que l'auteur traite des problèmes d'endogamie et d'exogamie.

(12) Claude Lévi-Strauss, op. cit., p. 83.

(13) Yvonne Verdier, Façons de dire, façons de faire. La laveuse, la couturière, la cuisinière, Paris, NRF Gallimard, 1979, p. 297, 298, 299. 


\subsection{Faire la cuisine}

Comme nous venons de le voir, Zéralda sait faire la cuisine c'est-à-dire qu'elle a le formidable pouvoir de réaliser des plats aux effets incroyables qui métamorphosent un ogre sanguinaire en mari aimant ! Elle n'est pas pour autant une sorcière ${ }^{(14)}$, tout au plus une fée... du logis car sa cuisine est vouée à une œuvre de vie humaine, humanisée, humanisante ${ }^{(15)}$. On le sait depuis Lévi-Strauss, la cuisine ${ }^{(16)}$ est une activité de transformation qui articule nature et culture : les lièvres du « saltus », nous l'avons montré, deviennent effectivement ici (dans notre album) de superbes pâtés en croûte. La cuisine facilite donc, au moins métaphoriquement, tout processus de transformation ${ }^{(17)}$ et permet le passage de la zone confuse des conduites ensauvagées à un monde où les rapports sociaux sont maîtrisés. Et c'est à la cuisinière Zéralda qu'incombe ce grand travail d'ordonnancement du monde dans l'univers fictionnel de l'album. Il n'y a qu'elle qui puisse occuper le rôle de passeuse.

Cela commence dès la première rencontre avec l'ogre. Ce dernier à l'affût comme une bête sauvage a senti l'odeur de la petite fille apportée par « un souffle de la brise matinale ». Il se précipite, tombe des rochers et s'effondre sur le chemin. «Sans connaissance», impuissant, il ne peut plus se conduire en « monstre affamé ». Quant à Zéralda, sa méconnaissance des ogres (ils n'existent que dans les contes) et, d'une certaine manière, son aptitude innocente à faire coexister le civilisé et le sauvage lui permettent d'être sensible à la part humaine de l'ogre (ce que n'avaient pas su faire les habitants de la ville) et donc de se porter à son secours pour le réintégrer immédiatement dans la communauté des hommes : «Oh, pauvre homme. » Elle l'initie aussitôt à l'art du cuit qu'elle sait décliner sous toutes ses formes. Lui qui pratique le cru n'a qu'une utilisation rudimentaire et limitée du cuit. Mais grâce au savoir de la fillette, les saveurs et les textures offertes se multiplient, les combinaisons et les associations de couleurs explosent dans les plats.

Les manières de table changent également. Zéralda qui peut défaire et déplacer les limites, installe une nappe blanche sur le chemin, y dispose un bouquet de fleurs et fait de ce « campus », un espace domestique. L'ogre allongé sur le flanc, mange alors en tenant délicatement la cuillère, le petit doigt tendu. La force socialisatrice de la cuisine parvient même à substituer à la solitude du goinfre des premières pages, la convivialité des banquets. Invités, les ogres amis apprécient à leur tour les mets délicats et se convertissent sans difficulté à un régime alimentaire «civilisé », même si leur acculturation n'est pas terminée. Les convives, l'image le montre, n'ont pas encore totalement abandonné leurs manières

(14) Effectivement, Zéralda n'est pas une sorcière même si l'ogre nous dit le texte est « enchanté » par ses talents culinaires. Il y a bien un balai prés de la cheminée mais ce n'est pas une monture, un chaudron est bien suspendu à la crémaillère, mais ce ne sont pas des ingrédients incongrus - rognures d'ongles, langues de crapauds — qui mijotent doucement.

(15) Yvonne Verdier, op. cit., p. 343. Claudine Fabre-Vassas, " La cuisine des sorcières ", Ethnologie française, 1991-4, Octobre-Décembre, tome 21, p. 423-437.

(16) Claude Lévi-Strauss, Mythologiques III. L'origine des manières de table, Paris, Plon, 1968.

(17) Yvonne Verdier, «Pour une ethnologie culinaire », op. cit., p. 54-55. 
« ogresques » : corsages et pourpoints ouverts, ceintures dégrafées, bouches ouvertes sur des rangées de grandes dents. Certains mangent encore avec les doigts...

\subsection{Cuisiner l'alphabet}

Le savoir et le pouvoir culinaires de l'héroïne sont toujours associés dans l'album de Tomi Ungerer à l'écrit. Notre Zéralda ne sait pas seulement faire la cuisine, elle cuisine aussi l'alphabet. Elle consulte les livres de recettes existants (on la voit lire dans la cuisine de la petite maison dans la clairière), elle remplit de ses nouvelles recettes « des livres et des livres de cuisine» nous dit le texte. Dès qu'elle compose un menu « extraordinaire », elle le transforme immédiatement en pages d'écriture. Dans le même mouvement, elle fait accéder l'ogre aux plaisirs conjugués de la cuisine et de la lecture. Il mange en lisant, il lit en mangeant. Les ogres du voisinage sont eux aussi invités à associer manger, lire et même écrire. Ils demandent à Zéralda de leur donner ses recettes et l'on voit une ogresse ravie noter sous la dictée les ingrédients nécessaires à la réalisation d'un plat savoureux. L'écrit et la cuisine sont donc de puissants adjuvants indissociables du processus de socialisation.

Effectivement l'ogre passe de pratiques appartenant à la culture orale à des pratiques fortement ancrées dans l'ordre de la littératie. Au début de l'album, par exemple, le personnage ne peut rassasier son immense appétit. Il profère des formulettes où se mêlent colère et plainte. Ces sortes d'incantations maléfiques sont sensées faire apparaître son plat préféré. Leur dimension ludique les rattache à la littérature enfantine orale, au corpus des comptines : «Craque et croque, si maintenant je rencontre quelques enfants, je les dévore à belles dents. » L'ogre qui, d'une certaine manière, se situe dans l'enfance de la socialisation, doit se détacher de l'oralité native pour accéder à l'ordre graphique.

Dans nos sociétés hautement alphabétisées, les effets de l'écrit sur le rapport à soi et au monde se pensent en termes de «domestication» de la pensée sauvage et de rationalisation des activités cognitives et sociales ${ }^{(18)}$. On peut dire alors que la métamorphose de l'ogre est due tout autant aux plats cuisinés par Zéralda qu'aux menus imprimés dont ils sont inévitablement accompagnés.

Le menu en lui-même appartient au genre de la liste et, en ce sens, il est une figure spécifique de l'écrit. Il suppose un certain agencement matériel, une certaine disposition spatiale, il comporte un début et une fin, il présente les aliments classés dans un ordre donné. Non seulement il procède à la mise en ordre des aliments, mais il les rend plus abstraits. Aussi Zéralda, en bonne pédagogue, a-telle toujours le souci d'associer le concret de la dégustation des plats à l'abstraction généralisante de leur mise en mots pour faire avancer l'ogre sur le chemin de la socialisation. Si l'incorporation des plats permet au mangeur d'être satisfait sans détour, la lecture du menu débouche plutôt sur une forme d'appropriation des codes qui donne accès à un savoir gastronomique (et à un certain savoir-vivre, comme dans les manuels de civilité).

(18) Jack Goody, Pouvoirs et savoirs de l'écrit, La Dispute, 2007, Présentation de J.-M. Privat, p. 11. 
Lire un menu, c'est donc s'approprier un vocabulaire culinaire, un goûteux technolecte. L'album propose deux menus rédigés, intéressants dans leur diversité. Le premier assez redondant est descriptif : il propose un équivalent verbal de ce qui se trouve dans l'assiette : " Potage de cresson à la crème. » C'est plutôt une sorte d'étiquetage. Le deuxième, par contre, montre l'inventivité de la cuisinière qui manie avec autant d'assurance les ingrédients que le langage. En utilisant dans la dénomination des plats, des reformulations métaphoriques où se mêlent l'humour et les références culturelles, elle crée une distance et fait aller beaucoup plus loin dans l'accession à l'ordre symbolique. Deux exemples sont particulièrement significatifs :

— «Dinde jeune fille» : l'appellation repose sur le nom de la volaille auquel est associé le stéréotype, « dinde », à prendre au sens de « tendre jeune fille un peu naïve ». La métaphore oblige à ne pas en rester au sens littéral, à ne pas prendre les mots au pied de la lettre, à cerner l'humour qui établit une sorte de pléonasme entre « dinde » et « jeune fille », bref à transformer les pulsions dévoratrices en signes comestibles.

— «Croque fillette sur délices des ogres », est le point d'aboutissement de ce travail métaphorique. La présence même de cette appellation indique que l'ogre a basculé dans le symbolique ; il est en passe de devenir un individu de plus en plus fréquentable qui ne confond plus les mots et les mets, le signe et son référent!

En effet, la pratique des écrits culinaires et de la métaphore gastronomique ont permis un glissement qui trouve son achèvement quelques pages avant la fin de l'album. On peut prendre alors la mesure de tout le chemin parcouru. Dans l'incipit, un ogre qui mange est mis en images et en mots. Mais au moment où le parcours de socialisation touche à sa fin, une double page montre le menu type « d'un souper tout à fait moyen au château du géant ». Ce qui est donné à voir et à lire en fait, c'est l'expression figurée « manger comme un ogre », même si elle ne se trouve pas inscrit telle quelle dans le texte. Et le passage à l'expression figée qui signifie manger énormément situe définitivement l'ogre et ses appétits hors normes humaines dans le seul univers qui est le sien désormais, le monde du langage, la logosphère.

\section{Conte de fées, conte de faim?}

\subsection{La clôture du texte}

L'explicit de l'album s'organise en fait en deux temps. Une première conclusion met en scène la fin de la faim. L'ogre peut retourner dans la ville maintenant que sa faim est rassasiée. Il ne met plus en danger la « domus ». Converti à la pratique du cuit et de l'écrit, il parvient à faire coexister en lui, la part ensauvagée et la part humanisée. Sa tenue vestimentaire s'est modifiée : bras et torse sont recouverts d'un pourpoint. Mais il a encore conservé sa grosse barbe. De leur côté, les habitants de la ville, rassurés, acceptent de voir en lui l'homme et plus seulement le monstre terrifiant. Il ne fait plus peur aux enfants. De Père Fouettard, no- 
tre ogre s'est quasiment transformé en Père Noël et même en futur père ${ }^{(19)}$. Une deuxième fin clôt définitivement le livre : «Les années passèrent... » nous dit le texte. Il faut en effet du temps pour devenir un individu parfaitement socialisé. Grandir nécessite de sortir du temps cyclique, le temps de la répétition où « chaque jour " l'ogre venait en ville pour dévorer des enfants, pour entrer dans le temps biographique et historique qui autorise les changements, les transformations et les évolutions.

Alors ce qui génériquement devait arriver arriva : «Ils devinrent amoureux l'un de l'autre. Ils se marièrent, etc. » Le travail de socialisation transforme les configurations culturelles : il fait passer de la triple homologie entre le cru, le corps soumis aux pulsions sexuelles et l'oralité à un autre système d'homologie qui établit un rapport entre le cuit, la sexualité maîtrisée c'est-à-dire la procréation et l'écrit.

\subsection{Le cycle des apprentissages}

Toutefois, avant de refermer le livre, il reste à observer attentivement cette image du bonheur que propose la dernière illustration de l'album et le texte qui l'accompagne. Certes la métamorphose est achevée : «L'ogre, toujours bien nourri, rasa sa barbe piquante. » Il a accompli le rite. L'homme sauvage de carnaval s'est dépouillé de sa sauvagerie animale, mais pas de son nom, on continue à l'appeler « l'ogre ». La fillette est devenue une jolie jeune femme qui a donné naissance à de nombreux enfants ${ }^{(20)}$, mais elle conserve son prénom énigmatique.

D'autres éléments évoquent le sauvage, à la lisière de l'image. C'est le château dont les tourelles rappellent le château du commencement et le temps où l'ogre sévissait. C'est le petit nœud rose qui entoure le cou du bébé et qui tout au long de l'album se trouvait autour du cou du chat. C'est enfin et surtout le couteau et la fourchette dissimulés dans le dos d'un des enfants qui signalent que la lignée des ogres n'est pas éteinte. La coexistence pacifiée du domestiqué et de l'ensauvagé est donc inscrite dans la dernière image, mais elle reste vacillante. Qui d'autre que Zéralda a donné le jour à ce petit monstre ! Les limites peuvent s'effacer, l'ensauvagement peut à nouveau s'imposer. En témoigne aussi l'ultime phrase du récit qui ouvre des possibles narratifs que le conte traditionnel referme généralement : «On peut donc penser que leur vie fut heureuse jusqu'au bout »

En renvoyant au début de l'histoire, cette fausse clôture indique que « toute limite est interrogée, défaite puis retracée provisoirement dans une action qu'il

(19) Aux enfants dévorés avec du sel et du poivre du début répondent les enfants sucrés léchant avec gourmandise les sucettes généreusement distribuées. Contrairement à l'assaisonnement qui était raté, le sucrage est réussi : cette fois les enfants ne disparaissent pas, ils sont nombreux à venir à la rencontre de l'ogre qui montre ainsi aux yeux de tous ses capacités à devenir père. Yvonne Verdier, Façons de dire, façons de faire, p. 298-299.

(20) «La statuaire saint-sulpicienne met en scène la transformation de la jeune fille par le sang menstruel (avoir ses fleurs) en utilisant des fleurs: Sainte Thérèse de l'Enfant Jésus est souvent représentée, environnée de roses, qu'elle offre à « l'époux divin. » Daniel Fabre, Une culture paysanne, Les formes de la culture, Histoire de la France, sous la direction d’André Brugière et Jacques Revel, Paris, Seuil, 1993, p. 167. 
faut (donc) sans cesse répéter ${ }^{(21)}$. » En insistant sur la répétition, le recommencement, la fin s'affiche comme une fin sans fin générale : chaque enfant devra parcourir, à son tour, le chemin qui mène à l'âge adulte.

Mais - conte oblige - chaque petit ogre rencontrera sur le chemin une Zéralda qui lui fera passer les limites du sauvage et le fera entrer dans le processus de civilisation. Alors le moment venu, l'un avec l'autre, ils pourront réaliser leur destin d'homme et de femme et fonder une famille.

Tout cela n'était-il pas d'ailleurs donné à lire dès le titre, dans le prénom crypté de l'héroïne ? Dans ce prénom étrange (ou tout au moins peu courant) — «Zéralda »- la lettre initiale et la lettre finale renversent l'ordre alphabétique et iconisent un parcours initiatique où les fins et les commencements s'enchaînent.

Et à y regarder de plus près, le seul qui voit vraiment que l'histoire n'est pas finie et va recommencer c'est le lecteur! Les regards de tous les personnages convergent sur le nouveau-né. Zéralda, les yeux baissés, presque clos, semble ne rien voir. Les personnages peuvent ne pas voir ou feindre de ne pas voir, le lecteur ne le peut pas!

Pour que le rituel soit totalement accompli, pour que le lecteur soit pleinement initié et devienne à son tour un initiateur, l'album doit lui ouvrir les yeux. Et c'est bien ce qu'assure le dévoilement de la dernière page. Si un rite biographique, par définition, ne se recommence pas, le récit littéraire du rite peut se recommencer pour soi ou pour les autres, autant de fois que nécessaire pour montrer le chemin et le cheminement. Et initier ainsi le jeune lecteur qui dévore les pages au rite de la lecture, à la pratique de la littérature « ogresque » et de ses rites domestiques.

(21) Daniel Fabre, «Limites non frontières du Sauvage », op. cit., p. 438. 


\section{Bibliographie}

BRAUdel, F. (1986) : L'Identité de la France. Espace et Histoire, Paris, ArthaudFlammarion.

BOURDIEU, P, (2000) : Esquisse d'une théorie de la pratique, précédé de trois études d'ethnologie kabyle, Paris, Editions du Seuil (première parution 1972).

DESCOLA, P. (2005) : Par-delà nature et culture, Paris, NRF, Editions Gallimard.

FABRE, D. (1993) : Une culture paysanne, Les Formes de la culture, Histoire de la France, sous la direction d'André Brugière et Jacques Revel, Paris, Editions du Seuil.

— (2005) : «Limites non frontières du Sauvage », L'Homme, n $175-176$ Vérités de la fiction, Paris, Editions de l'EHESS.

Goody, J. (2007) : Pouvoirs et savoirs de l'écrit, Paris, La Dispute, Présentation de J.-M. Privat.

LÉVI-S TRAUSS, C. (1968) : Mythologiques III. L'Origine des manières de table, Paris, Plon.

- (1983) : Le Regard éloigné, Paris, Plon.

LE ROY LADURIE, E. (1973) : «La domus à Montaillou », Ethnologie Française III, $\mathrm{n}^{\circ} 1-2$.

VERdiER, Y. (1969) : «Pour une ethnologie culinaire », L'Homme, n 1 . Paris, Editions de l'EHESS.

- (1979) : Façons de dire, façons de faire. La laveuse, la couturière, la cuisinière, Paris, NRF, Editions Gallimard.

- (1995) : Coutume et Destin. Thomas Hardy et autres essais, Paris, NRF, Editions Gallimard. 